\title{
Study of GBP2 Gene Expression and Its Promoter Methylation Pattern in Tumors of Breast Cancer Patients
}

\author{
Farzaneh Rahvar ${ }^{1}$, Mahdieh Salimi ${ }^{2,}$, Hossein Mozdarani ${ }^{3}$ \\ ${ }^{1}$ Research and technology department, Islamic Azad University, Iran \\ ${ }^{2}$ Department of Medical Genetics, Institute of Medical Biotechnology, National \\ Institute of Genetic Engineering and Biotechnology (NIGEB), Tehran, Iran \\ ${ }^{3}$ Department of Medical Genetics, Tarbiat Modares University, Tehran, Iran \\ * Corresponding author: Mahdieh Salimi, Department of Medical Genetics, Institute \\ of Medical Biotechnology, National Institute of Genetic Engineering and Biotechnology \\ (NIGEB), Tehran, Iran.E-mail: salimi@nigeb.ac.ir
}

DOI: $10.21859 / \mathrm{mci}$-supp-04

\author{
Keywords: \\ Breast Cancer \\ Promoter Methylation \\ Gene Expression \\ GBP2 Gene \\ Epigenetics \\ Cancer Biomarker
}

\begin{abstract}
Introduction: Breast cancer is the most common invasive cancer in women around the world. Guanylate binding protein 2 (GBP2) is a member of GBP family. Interferon-inducible guanylate binding protein (GBP2) has been discussed as a possible control factor in tumor development in breast cancer that have potential to be a tumor marker.

Materials and Methods: In this study, SYBR green Real Time RT-PCR was used to examine expression of GBP2 gene in tumoral tissues in comparison with normal ones, in presence of a housekeeping gene as an internal control in 60 breast cancer patients. Furthermore, possible changes of GBP2 promoter methylation pattern was analyzed using MS-PCR technique.

Results: Our results indicated that GBP2 gene expression and its promoter methylation pattern in normal tissues and tumoral tissues were significantly different. The GBP2 gene was down regulated in tumor tissues compared with normal adjacent breast tissues and the promoter area of this gene was hyper methylated in tumoral tissues.

Conclusions: As a consequence, GBP2 expression and its promoter methylation pattern are associated with breast cancer as possible biomarker. Also GBP2 promoter methylation pattern may influence its expression and affects cancer pathologic characteristics. More information in this field may be promising in cancer management.
\end{abstract}

